\title{
Role of MiR-205/PTEN in cisplatin-resistant esophageal squamous cell carcinoma
}

\author{
Xun Tang ${ }^{1-3}$, Xuelian Mao ${ }^{1-3}$, Feng Yan ${ }^{1-3 *}$ \\ ${ }^{1}$ Jiangsu Cancer Hospital, ${ }^{2}$ Jiangsu Institute of Cancer Research, ${ }^{3}$ Department of Clinical Laboratory, The Affiliated Cancer \\ Hospital of Nanjing Medical University, Nanjing, 210009, China
}

*For correspondence: Email: yanfengjiop@163.com

\begin{abstract}
Purpose: To evaluate the effect of miRNA-205 on cisplatin-resistant TE13 cell lines, and the interaction of miRNA-205 with downstream signaling pathway.

Methods: TE13 cells were treated with cisplatin at a concentration of $1.5 \mu \mathrm{g} / \mathrm{mL}$ every $24 \mathrm{~h}$ for 3 months. Cisplatin-resistant cell lines were maintained in the continuous presence of $2 \mu \mathrm{g} / \mathrm{ml}$ cisplatin and supplemented every $72 \mathrm{~h}$. Half-maximal inhibitory concentration $\left(I C_{50}\right)$ value was determined by MTT assay, and cell apoptosis was tested by flow cytometry. In addition, luciferase reporter assay was conducted to test the binding of miRNA-205 and phosphatase and tensin homologue deleted on chromosome 10 (PTEN) 3'UTR.

Results: The cisplatin-resistant (cis-TE13) cell line was well established based on IC $C_{50}$ values (cisTE13, IC $C_{50}=2.215$; TE13, IC $C_{50}=0.304$ ). The levels of miRNA-205, phosphatase, PTEN, and protein kinase B (p-AKT) were abnormally expressed in cis-TE13 cells. Overexpression of miRNA-205 significantly promoted cell viability and decreased cell apoptosis of cis-TE13 cells as indicated by IC $C_{50}$ values (cis-TE13, IC $C_{50}=3.537 ;$ TE13, IC $C_{50}=0.580$ ). Moreover, miRNA-205 significantly activated $p$-AKT expression by inhibiting PTEN expression. In contrast to miRNA-205, PTEN overexpression inhibited cell viability, increased cell apoptosis of cis-TE13 cells (cis-TE13, IC $C_{50}=2.625$; TE13, IC $C_{50}=0.246$ ), decreased the expression of $p-A K T$, and counteracted the regulation of miRNA-205 (control, IC $C_{50}=$ 2.297; $m i R-205, I C_{50}=4.693 ;$ miR-205+PTEN, IC $50=2.011$ ).

Conclusion: These findings indicate that miRNA-205 exacerbates esophageal squamous cell carcinoma via inhibition of PTEN expression, suggesting the potential value of miRNA-205 in the diagnosis or treatment of esophageal squamous cell carcinoma.
\end{abstract}

Keywords: miRNA-205, PTEN, PI3K/AKT signaling pathway, Esophageal squamous cell, Carcinoma, Cisplatin resistance

\begin{abstract}
This is an Open Access article that uses a funding model which does not charge readers or their institutions for access and distributed under the terms of the Creative Commons Attribution License (http://creativecommons.org/licenses/by/4.0) and the Budapest Open Access Initiative (http://www.budapestopenaccessinitiative.org/read), which permit unrestricted use, distribution, and reproduction in any medium, provided the original work is properly credited.

Tropical Journal of Pharmaceutical Research is indexed by Science Citation Index (SciSearch), Scopus, International Pharmaceutical Abstract, Chemical Abstracts, Embase, Index Copernicus, EBSCO, African Index Medicus, JournalSeek, Journal Citation Reports/Science Edition, Directory of Open Access Journals (DOAJ), African Journal Online, Bioline International, Open-J-Gate and Pharmacy Abstracts
\end{abstract}

\section{INTRODUCTION}

Esophageal adenocarcinoma is believed to be the most rapidly increasing cancer in Western countries. However, esophageal squamous cell carcinoma is the most common digestive system malignancy in Asia [1]. Although chemotherapy has been widely used in the clinical treatment of esophageal squamous cell carcinoma, unfortunately, the resistance to chemotherapy 
drugs, especially cisplatin chemoresistance, occurs in a large proportion of patients. [2,3]. Therefore, exploring the molecular mechanisms responsible for the cisplatin chemoresistance of esophageal squamous cell carcinoma cells is of great significance to improve the therapeutic efficacy of cisplatin. The small non-coding regulatory RNAs, microRNAs (miRNAs) target numerous tissues and cells, including the nervous and immune systems. These molecules are involved in many biological and pathologic processes and function as oncogenes or tumor suppressors [4]. The miRNA-205 is a suppressor for many tumors such as breast cancer and prostate carcinoma [5]. However, the expression of miRNA-205 varies between tumors and remains controversial. Therefore, the expression of miRNA-205 and its downstream signaling pathway should be further studied in different cancers.

According to previous research, PTEN belongs to a family of inhibitory factors for tumors and can be regulated by miRNA-205 in many kinds of tumors. Moreover, the PI3K/AKT signaling pathway participates in the modulation of tumor development with miRNA-205 and PTEN[6]. In this study, the expression of miRNA-205 and PTEN in cisplatin-resistant esophageal squamous cell carcinoma cell lines was investigated. Furthermore, miRNA-205 was identified as a positive regulator of AKT signaling by targeting PTEN for downregulation.

\section{EXPERIMENTAL}

\section{Establishment of cisplatin-resistant cell lines}

TE13 cells were seeded in 6-well plates in Roswell Park Memorial Institute-1640 medium (RPMI-1640; Gibco, Grand Island, NY) and were treated with cisplatin at a concentration of 1.5 $\mu \mathrm{g} / \mathrm{mL}$ every $24 \mathrm{~h}$ for 3 months. Before the experiment, the cis-TE13 cells were treated at a concentration of $1.5 \mathrm{ug} / \mathrm{mL}$ for three cycles, each lasting $72 \mathrm{~h}$. Then, the cells were treated with cisplatin at a concentration of $2 \mu \mathrm{g} / \mathrm{mL}$ for three cycles, each lasting 72 h. Cisplatin-resistant cell lines were maintained in the continuous presence of $2 \mu \mathrm{g} / \mathrm{mL}$ cisplatin, which was supplemented every $72 \mathrm{~h}$. The $\mathrm{IC}_{50}$ values were used to evaluate the resistant cell model. The cells were grouped into two: TE13 (control) and cis-TE13 (cisplatin-resistant TE13 cells) [7].

\section{Overexpression and knockdown of miRNA- 205 and PTEN in TE13 cells}

The cis-TE13 cells were transfected with empty pCMV6 vector, pCMV6 with a sequence encoding for miRNA-205, empty pGEX-6p-1 vector, or pGEX-6p-1 with sequence encoding for PTEN (ORIGENE, Rockville, MD) using FuGENE HD (Roche, Shanghai, China). The oligonucleotide fragments of negative control (siNC-in) and siRNA targeting miRNA-205 (simiRNA-205) were purchased from Genephama Biotech (Shanghai, China) for the knockdown of miRNA-205 using FuGENE HD.

\section{Reverse transcription-quantitative polymerase chain reaction (RT-qPCR)}

Total RNA from TE13 and cis-TE13 cells was isolated using Trizol Reagent (Invitrogen). Then, the RNA was reverse transcribed into cDNA using SuperScript reverse transcriptase (ThermoFisher, Cheshire, UK). To test the transfection efficiency of miR-205 and PTEN, RT-PCR analysis was performed with primers for miR-205 and PTEN (Table 1). The mRNA levels of miRNA-205 and PTEN were normalized with U6snRNA obtained with primers for U6 (Table 1).

\section{Western blot assay}

Whole cell lysates from TE13 cells following overexpression and knockdown of miRNA-205 and PTEN were extracted on ice with a solution containing Tris- $\mathrm{HCl}(20 \mathrm{mM}, \mathrm{pH}=7.5)$, Triton $\mathrm{X}$ 100 (1\%), and PMSF (1\%). After protein quantitation, the proteins were separated by 10 $\%$ SDS-PAGE and were transferred on to PVDF membranes, which were blocked with $5 \%$ non-fat milk. Then the membranes were probed with antibodies against PTEN, AKT, p-AKT, or GAPDH (Abcam, Cambridge, MA) for $16 \mathrm{~h}$ at 4 ${ }^{\circ} \mathrm{C}$. Then, the membranes were incubated with secondary anti-goat and anti-mouse antibody for $2 \mathrm{~h}$ at $37^{\circ} \mathrm{C}$.

\section{Luciferase reporter assay}

The binding of miRNA-205 to PTEN 3'UTR was tested using a luciferase reporter assay.

Table 1: Primer sequences for RT-qPCR

\begin{tabular}{lll}
\hline Gene & Forward primer & Reverse Ppimer \\
\hline miR-205 & 5'-CTT GTC CTT CAT TCC ACC GGA-3' & 5'-TGC CGC CTG AAC TTC ACT CC-3' \\
PTEN & 5'-TGT GGT CTG CCA GCT AAA GG-3' & 5'-CGG CTG AGG GAA CTC AAA GT-3' \\
U6 & CTCGCTTCGGCAGCACA & AACGCTTCACGAATTTGCGT \\
\hline
\end{tabular}


As previously described, the entire 3'-UTR of human PTEN was cloned into the pGL3promoter vector containing luciferase. The PTEN 3'UTR sequences were mutated at the miRNA205 binding sites using a QuikChange II SiteDirected Mutagenesis kit (Stratagene, La Jolla, CA). For this assay, TE13 cells were plated in 24-well plates and transfected with pGL3promoter vector using FuGENE HD.

\section{Flow cytometry assay}

A Calibor ${ }^{\mathrm{TM}}$ cytometer (BD, American) was used to measure the DNA content and cell apoptosis with ModFit LTTM software. The TE13 cells were collected $45 \mathrm{~h}$ after transfection. Pancreatic enzymes with no EDTA were used for cell dissociation. Cells were washed with PBS and were resuspended in alcohol $\left(70 \%, 4{ }^{\circ} \mathrm{C}\right)$ followed by the addition of PI/RNase staining buffer solution (Abcam) for incubation overnight at $4{ }^{\circ} \mathrm{C}$. After that, the solution was replaced with $500 \mu \mathrm{L}$ PI/RNase staining buffer solution. In each sample, 20,000 events were analyzed.

\section{Cellular viability assay}

A total of $2 \times 10^{6}$ TE13 cells were seeded in 6well plates in DMEM medium and grown in the normal environment. After $36 \mathrm{~h}$, the cells were washed with PBS (Gibco), incubated with DAPI (Abcam) for $30 \mathrm{~min}$, and then observed under a microscope.

\section{CCK8 assay}

A cell-counting kit (CCK8) assay was also conducted to test cell proliferation. Cells $\left(1 \times 10^{5}\right)$ were plated in 96-well plates and incubated with CCK-8 solutions (R\&D Systems, Minneapolis, $\mathrm{MN})$ ) for $1.5 \mathrm{~h}$ at $37^{\circ} \mathrm{C}$.

\section{Statistical analysis}

All values are presented as mean \pm standard deviation (SD) and were analyzed with GraphPad 7.0 and SPSS 18.0. Student's $t$-test was used to compare two groups, and one-way ANOVA and Dunnett test were used to compare several groups with the control. Two-tailed $p$ values $<0.05$ were considered statistically significant.

\section{RESULTS}

Aberrant expression of miRNA-205, PTEN,
and PI3K/AKT signaling in cis-TE13 cells

Cisplatin-resistant cells were well-established as indicated by the difference in cell apoptosis between the TE13 cell line and the cis-TE13 cell line. The $\mathrm{IC}_{50}$ value for the TE13 cell line was 0.304 , while the value for cis-TE13 was 2.215 (Figure $1 \mathrm{~A}$ ). Results showed that the levels of miRNA-205 were increased in cis-TE13 cells (Figure $1 \mathrm{~B}$ ). The protein level of PTEN was decreased in cis-TE13 cells; however, the protein level of $\mathrm{p}$-AKT was increased in cis-TE13 cells (Figure $1 \mathrm{C}$ ). The protein level of AKT was not changed.
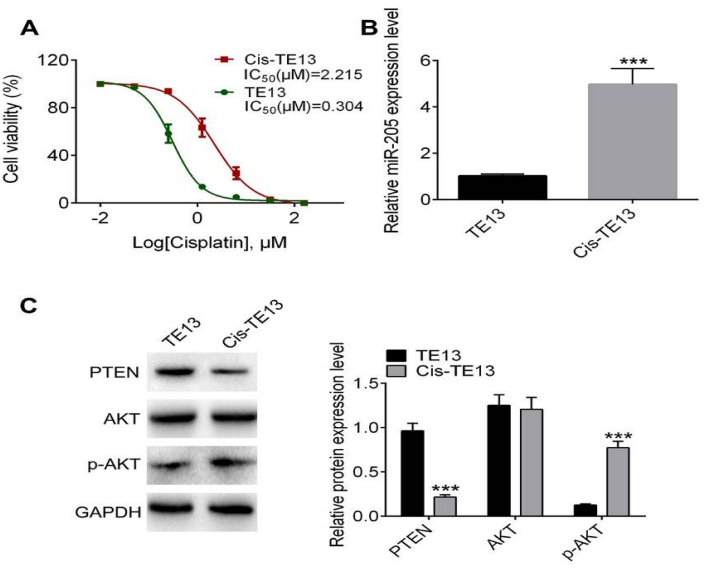

Figure 1: Aberrant expression of miRNA-205, PTEN, and PI3K/AKT signaling in cisplatin-resistance TE13 cells. (A) Cell viability and $\mathrm{IC}_{50}$ values of TE-13 and cis-TE13 cells. (B) The mRNA level of miRNA-205 in cis-TE13 and TE13 cells. (C) Protein levels of PTEN, AKT, and p-AKT in cis-TE13 and TE13 cells. $\mathrm{N}=10$; ${ }^{* * *} p<0.001$ versus control, as determined by unpaired Student's $t$-test and one-way ANOVA analysis

\section{Enhanced resistance to cisplatin by knockdown of miRNA-205 gene in cis-TE13 cells}

To further investigate the effect of miRNA-205 on esophageal squamous cell carcinoma, we conducted miRNA-205 knockdown and qRTPCR analysis. Results showed that miRNA-205 knockdown decreased the expression of miRNA205 in cis-TE13 cells (Figure 2 A). After miRNA205 knockdown, the cis-TE13 cells were incubated with different concentrations of cisplatin. Compared with the control group, the knockdown of miRNA-205 decreased the viability of cis-TE13 cells in the presence of cisplatin (Figure $2 \mathrm{~B}$; NC-in, $\mathrm{IC}_{50}=3.537$; miR-205-in, $\mathrm{IC}_{50}$ $=0.580$ ) and increased cell apoptosis (Figure 2 C).

\section{Modulation of PTEN and PI3K/AKT signaling by miRNA-205 in cis-TE13 cells}

Above results showed that cis-TE13 cells expressed higher level of miRNA-205 and lower level of PTEN than TE13 cells. Therefore, a luciferase reporter assay was performed to study 
the interaction between miRNA-205 and the PTEN gene. Figure $3 \mathrm{~A}$ shows the sequence of PTEN 3'UTR and Mut and Wt miRNA-205 in the luciferase reporter assay. Figure $3 \mathrm{~B}$ shows an increase in mRNA levels of miRNA-205 in cisTE13 cells following miRNA-205 overexpression. Luciferase reporter assay results showed that the expression levels of fluorescent protein in the miRNA-205 overexpression group of PTEN 3'UTR-Wt cells were lower than that in the control group of PTEN 3'UTR-Wt.

A

C

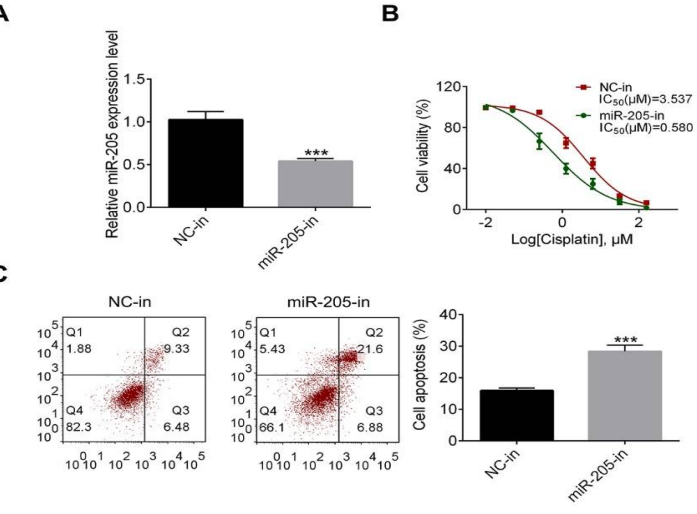

(control, $\mathrm{IC}_{50}=2.625 ;$ PTEN overexpression, $\mathrm{IC}_{50}$ $=0.246$ ). Overexpression of PTEN also increased the protein levels of PTEN and decreased the protein levels of p-AKT (Figure $4 C)$. The AKT protein level was not changed by PTEN overexpression (Figure 4C). Additionally, flow cytometry results further confirmed that PTEN overexpression increased cell apoptosis (Figure 4D).

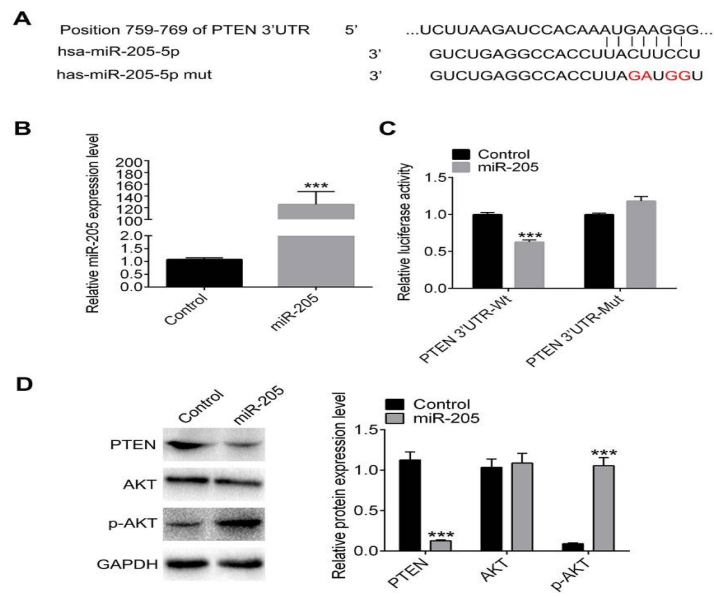

Figure 3: Modulation of PTEN and PI3K/AKT signaling by miRNA-205 in cisplatin-resistant TE13 cells. (A) Sequence of the PTEN 3'UTR and miRNA205 of Mut and Wt for the luciferase reporter assay. (B) mRNA levels of miRNA-205 in control and miRNA205 overexpression cells. (C) Expression level of fluorescent protein in cis-TE13 and control cells cotransfected with miRNA-205 and either PTEN 3' UTRWt or -Mut. (D) protein levels of PTEN, AKT, and pAKT in control and miRNA-205 overexpression cells. $n$ $=10 .{ }^{* *} p<0.001$, versus control by unpaired Student's $t$-test and one-way ANOVA

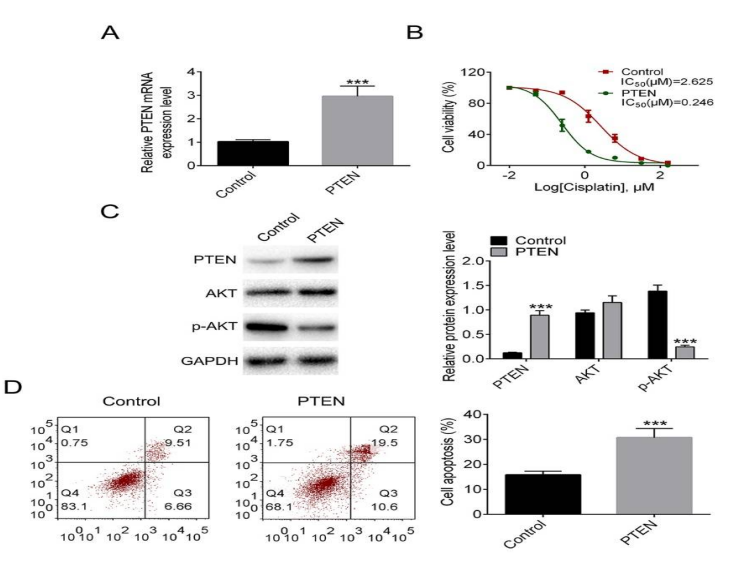

Figure 4: Improvement of resistance to cisplatin by overexpression of PTEN in cisplatin-resistant TE13 cells. (A) mRNA level of PTEN of control and PTEN overexpression cis-TE13 cells. (B) Cell viability of control and PTEN overexpression cis-TE13 cells. (C) The protein level of PTEN, AKT, and p-AKT of control and PTEN overexpression cis-TE13 cells. (D) Apoptosis of control and PTEN overexpression cisTE13 cells; $\mathrm{n}=10 ;{ }^{* *} p<0.01,{ }^{* *} p<0.001$, versus 
control by unpaired Student's $t$-test and one-way ANOVA

\section{Modulation of resistance to cisplatin by miRNA-205 and PTEN co-overexpression in cis-TE13 cells}

Based on the results above, cis-TE13 cells were co-transfected with miRNA-205 and PTEN overexpression plasmids to investigate the modulation of the PI3K/AKT signaling pathway by miRNA-205 and PTEN in cis-TE13 cells, using miRNA mimics as a control. Then, these cells were incubated with different concentrations of cisplatin. Results showed that miRNA-205 overexpression decreased the sensitivity of cisTE13 cells to cisplatin, while the cooverexpression of miRNA-205 and PTEN restored the sensitivity of cis-TE13 cells to cisplatin (Figure $5 \mathrm{~A}$ ), as indicated by the $\mathrm{IC}_{50}$ values (control, $\mathrm{IC}_{50}=2.297 ;$ miR-205, $\mathrm{IC}_{50}=$ 4.693; miR-205 + PTEN, $\quad \mathrm{IC}_{50}=2.011$ ). Compared with controls, the overexpression of miRNA-205 inhibited PTEN protein expression and increased the protein level of p-AKT. However, the co-overexpression of miRNA-205 and PTEN restored the protein levels of $p$-AKT and PTEN compared with control (Figure $5 \mathrm{~B}$ ). Consistent with the above results, miRNA-205 overexpression decreased cell apoptosis; however, co-overexpression of PTEN counteracted the effect of miRNA-205 overexpression, inducing the opposite effect of PTEN on cis-TE13 cells and the PI3K/AKT signaling pathway (Figure $5 \mathrm{C}$ ).

A
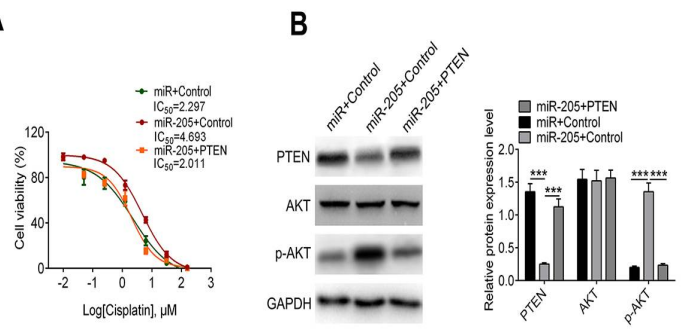

C

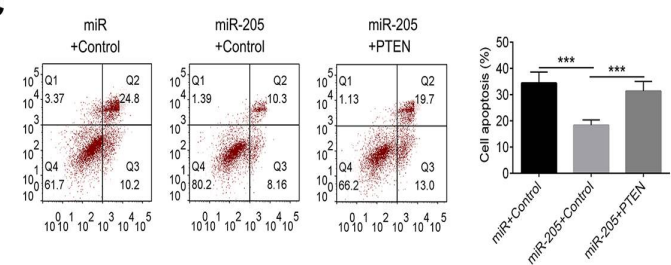

Figure 5: Modulation of resistance to cisplatin by miRNA-205 and PTEN co-overexpression in cisplatinresistance TE13 cells. (A) Viability of cis-TE13 cells transfected with PTEN and miRNA-205, control and miRNA-205, and control and miRNA control. (B) Protein levels of PTEN, AKT, and p-AKT in the cisTE13 cells transfected with PTEN and miRNA-205, control and miRNA-205, and control and miRNA control. (C) Apoptosis of cis-TE13 cells transfected with PTEN and miRNA-205, miRNA-205, and miRNA control; $\mathrm{n}=10 ;{ }^{* *} p<0.01,{ }^{* * *} p<0.001$, versus control by unpaired Student's $t$-test and one-way ANOVA

\section{DISCUSSION}

The current study demonstrated a carcinogenic and drug-resistance effect of miRNA-205. The first finding was aberrant expression of miRNA205 and the downstream proteins PTEN and PISK/AKT signaling pathway members in cisplatin resistant esophageal squamous cell carcinoma cells, consistent with a previous report[8]. It has been reported that the expression of miRNA-205 in esophageal squamous cell carcinoma clinical tissues was higher than in corresponding adjacent noncancerous tissue samples and that the expression was related to cancer stage, indicating that miRNA-205 is involved in the initiation and progression of esophageal squamous cell carcinoma [8].

In this study, miRNA-205 knockdown in esophageal squamous cell carcinoma cells had anti-tumor effects, including decreased viability and increased cell apoptosis of cis-TE13 cells. Furthermore, miRNA-205 transcriptionally inhibits the expression of PTEN and consequently increases the expression of PI3K/AKT signaling pathway protein $\mathrm{p}$-AKT. This result is consistent with results in lung cancer, in which PTEN is a target gene of miRNA-205 in A549 cells [9]. In addition, PTEN may act as an anti-tumor factor and directly decrease the expression of p-AKT. Moreover, co-transfection of miRNA-205 and PTEN demonstrated that PTEN may be an important node in the pathway involving miRNA205 and $\mathrm{p}-\mathrm{AKT}$.

miRNA-205 influences many cancers as an inhibitory factor that functions via different signaling pathways. For example, miRNA exerts a suppressive role in human prostate cancer, as miRNA-205 plays a direct inhibitory role on the epithelial-to-mesenchymal transition through downregulation of the protein kinase $C$ [10]. These results were first reported in 2009, and in 2014 , the same laboratory reported that miRNA205 influences the tumor microenvironment by preventing and reverting the oxidation in the process of the epithelial-to-mesenchymal transition [11]. Besides, miRNA-205 targets the E-cadherin transcriptional repressors ZEB1 and ZEB2 (also known as SIP1) to prevent the TGF$\beta$-induced epithelial-to-mesenchymal transition [12].

Trop J Pharm Res, December 2018; 17(12): 2351 
Furthermore, the E2F1 transcription factor is another target protein, and miRNA-205 is reported to inhibit anti-cancer chemotherapy resistance through inhibition of the E2F1 transcription factor [13]. Accordingly, it is well known that miRNA-205 is an anti-tumor factor for many cancers. However, there are also some studies that discuss contrary findings that miRNA-205 has high expression in some kinds of cancers, such as laryngeal squamous cell carcinoma [14]. Strikingly, it is reported that miRNA-205 may be an esophageal squamous cell carcinoma-specific miRNA that exerts tumorsuppressive activities through regulation of zinc finger E-box binding homeobox 2 expression in esophageal squamous cell carcinoma cells [15].

A possible explanation for this inconsistency is that different cell lines were used. The gene expression profile of cisplatin-resistant cancer cells may be different from that of normal cancer cells [16]. Therefore, another normal control group was needed in the future study.

PTEN may also be the important anti-tumor factor in this cancer type. The cisplatin resistance-related effect of PTEN has been shown in previous studies in which PTEN inhibited the chemoresistance to cisplatin in epithelial ovarian cancer [17]. MiRNA-205mediated PTEN downregulation is the key factor in chemoresistance of non-small cell lung cancer[18]. In non-small cell lung cancer, miRNA-205 was also reported to target PTEN and PHLPP2 for downregulation, thereby enhancing the proliferation of cancer cells [9].

However, in this study, the expression of AKT was not changed in cis-TE13 cells and not influenced by miRNA-205 or PTEN overexpression in cisplatin-resistant cells, while p-AKT expression was increased by miRNA-205 overexpression and decreased by PTEN overexpression in cis-TE13 cells. Furthermore, the results in this study showed that p-AKT was a downstream factor of PTEN. In addition to PTEN/p-AKT signaling pathway, TGF- $\beta$ signaling pathway also regulates miRNA-205 expression [19], suggesting a possible interaction between different cell signaling pathways.

\section{CONCLUSION}

Aberrant overexpression of miRNA-205 has been demonstrated in cisplatin-resistant esophageal squamous cell carcinoma cell lines. Further, it was shown that miRNA-205 promoted cell viability and decreased cell apoptosis of esophageal squamous cell carcinoma and activated the PI3K/AKT signaling pathway by inhibiting PTEN expression. Moreover, PTEN overexpression counteracted the miRNA-205 regulation. These findings indicate that miRNA205 may promote the resistance of esophageal squamous cell carcinoma cell lines to cisplatin via targeting of the PTEN gene. Thus, this molecule may be further investigated as an important target for the treatment of esophageal squamous cell carcinoma.

\section{DECLARATIONS}

\section{Acknowledgement}

This study was funded by National Natural Science Foundation of China (grant no. 201475063), State Key Laboratory of Analytical Chemistry for Life Science (grant no. SKLACLS1809) and program of Jiangsu Cancer Hospital (grant no. ZQ201502).

\section{Disclosure of interest}

The authors declare that no conflict of interest is associated with this work.

\section{Contribution of authors}

We declare that this work was done by the authors named in this article and all liabilities pertaining to claims relating to the content of this article will be borne by the authors. Xun Tang designed all the experiments and revised the manuscript. Xuelian Mao performed the experiments, Feng Yan wrote the manuscript.

\section{REFERENCES}

1. Mathé EA, Nguyen GH, Bowman ED, Zhao Y, Budhu A, Schetter AJ, Braun $R$, Reimers $M$, Kumamoto $K$, Hughes D. MicroRNA expression in squamous cell carcinoma and adenocarcinoma of the esophagus: associations with survival. Clin Cancer Res. 2009; 15(19): 6192-6200.

2. Ikeguchi M, Kohno $Y$, Kihara $K$, Suzuki $K$, Endo $K$, Nakamura S, Sawada T, Shimizu T, Matsunaga T, Fukumoto Y. Neoadjuvant Chemotherapy for Clinical Stage II and III Thoracic Esophageal Squamous Cell Carcinoma with Curative Esophagectomy. J Cancer Ther. 2015; 06(15): 1207-1213.

3. Tiwari S, Goel V, John MC, Patnaik N, Doval DC. Efficacy and toxicity of cetuximab with chemotherapy in recurrent and metastatic head and neck cancer: $A$ prospective observational study. Ind J Cancer. 2016; 53(4): 487-491.

4. Tsuchiya S, Okuno $Y$, Tsujimoto G. MicroRNA: biogenetic and functional mechanisms and involvements 
in cell differentiation and cancer. J Pharmacol Sci. 2006; 101(4): 267-270.

5. Hu Y, Qiu Y, Yagüe E, Ji W, Liu J, Zhang J. miRNA-205 targets VEGFA and FGF2 and regulates resistance to chemotherapeutics in breast cancer. Cell Dea Dis. 2016; 7(6): e2291.

6. Wang D, Wang S, Liu Q, Wang M, Wang C, Yang H. SZ$685 \mathrm{C}$ exhibits potent anticancer activity in both radiosensitive and radioresistant NPC cells through the miR-205-PTEN-Akt pathway. Oncol Rep. 2013; 29(6): 2341-2347.

7. Xu Y, Huang J, Ma L, Shan J, Shen J, Yang Z, Liu L, Luo Y, Yao C, Qian C. MicroRNA-122 confers sorafenib resistance to hepatocellular carcinoma cells by targeting IGF-1R to regulate RAS/RAF/ERK signaling pathways. Cancer Lett. 2016; 371(2): 171-181.

8. Liang B, Wu $Y$, Han $X$, Zheng $X$, Zhan $Q$, Tong T. MiR205 inhibits the invasion and migration of esophageal squamous cell carcinoma by modulating SMAD1 expression. Sci Bullet. 2014; 59(19): 2232-2239.

9. Cai J, Fang L, Huang Y, Li R, Yuan J, Yang Y, Zhu X, Chen B, WU J, Li M. miR-205 targets PTEN and PHLPP2 to augment AKT signaling and drive malignant phenotypes in non-small cell lung cancer. Cancer Res. 2013; 73(17): 5402-5415.

10. Gandellini $P$, Folini $M$, Longoni $N$, Pennati M, Binda $M$, Colecchia M, Salvioni R, Supino R, Moretti R, Limonta $P$ et al. miR-205 Exerts tumor-suppressive functions in human prostate through down-regulation of protein kinase Cepsilon. Cancer Res. 2009; 69(6): 2287-2295.

11. Gandellini P, Giannoni E, Casamichele A, Taddei ML, Callari M, Piovan C, Valdagni R, Pierotti MA, Zaffaroni $N$, Chiarugi $P$. miR-205 hinders the malignant interplay between prostate cancer cells and associated fibroblasts. Antioxid Redox Signal. 2014; 20(7): 10451059.

12. Gregory PA, Bert AG, Paterson EL, Barry SC, Tsykin A, Farshid G, Vadas MA, Khew-Goodall $Y$, Goodall GJ.
The miR-200 family and miR-205 regulate epithelial to mesenchymal transition by targeting ZEB1 and SIP1. Nat Cell Biol. 2008; 10(5): 593-601.

13. Lai X, Gupta SK, Schmitz U, Marquardt S, Knoll S, Spitschak A, Wolkenhauer O, Putzer BM, Vera J. MiR205-5p and miR-342-3p cooperate in the repression of the E2F1 transcription factor in the context of anticancer chemotherapy resistance. Theranostics. 2018; 8(4): 1106-1120.

14. Wang B, Lv K, Chen W, Zhao J, Luo J, Wu J, Li Z, Qin H, Wong TS, Yang $W$ et al. miR-375 and miR-205 Regulate the Invasion and Migration of Laryngeal Squamous Cell Carcinoma Synergistically via AKTMediated EMT. Biomed Res Int. 2016; 9652789(10): 19.

15. Matsushima $K$, Isomoto $H$, Yamaguchi $N$, Inoue $N$, Machida H, Nakayama $T$, Hayashi $T$, Kunizaki $M$, Hidaka S, Nagayasu $T$ et al. MiRNA-205 modulates cellular invasion and migration via regulating zinc finger E-box binding homeobox 2 expression in esophageal squamous cell carcinoma cells. J Transl Med. 2011; 9(30): 1479-5876.

16. Gosepath EM, Weykam S, Gruenewald E, Ko Y, Wiese $M$, Kassack MU. Differential gene expression studies in cisplatin-sensitive versus cisplatin-resistant human cancer cell lines. Int J Clin Pharmacol Ther. 2004; 42(11): 637-639.

17. Yu X, Chen Y, Tian R, Li J, Li H, Lv T, Yao Q. miRNA-21 enhances chemoresistance to cisplatin in epithelial ovarian cancer by negatively regulating PTEN. Oncol Lett. 2017; 14(2): 1807-1810.

18. Lei L, Huang Y, Gong W. miR-205 promotes the growth, metastasis and chemoresistance of NSCLC cells by targeting PTEN. Oncol Rep. 2013; 30(6): 2897-2902.

19. Duan Y, Chen Q. TGF-beta1 regulating miR-205/miR-195 expression affects the TGF-beta signal pathway by respectively targeting SMAD2/SMAD7. Oncol Rep. 2016; 36(4): 1837-1844. 